\title{
Reliability Constrained Unit Commitment Considering the Effect of DG and DR Program
}

\author{
Masoud Aliakbari ${ }^{1}$, Pouria Maghouli ${ }^{2}$, Habib Allah Aalami ${ }^{3}$ \\ ${ }^{1}$ Department of electrical engineering, Islamic Azad University, Saveh, Iran \\ ${ }^{2}$ Department of electrical engineering, Shahed University, Tehran, Iran \\ ${ }^{3}$ Department of electrical engineering, Eyvanekey University, Tehran, Iran
}

\begin{tabular}{l} 
Article Info \\
\hline Article history: \\
Received Jun 26, 2017 \\
Revised Jan 19, 2018 \\
Accepted Jan 26, 2018 \\
\hline
\end{tabular}

Keyword:

Demand response program

Distributed energy resources

Operation

Particle swarm optimization

Reliable and economic

Simulated annealing

Unit commitment

\begin{abstract}
Due to increase in energy prices at peak periods and increase in fuel cost, involving Distributed Generation (DG) and consumption management by Demand Response (DR) will be unavoidable options for optimal system operations. Also, with high penetration of DGs and DR programs into power system operation, the reliability criterion is taken into account as one of the most important concerns of system operators in management of power system. In this paper, a Reliability Constrained Unit Commitment (RCUC) at presence of time-based DR program and DGs integrated with conventional units is proposed and executed to reach a reliable and economic operation. Designated cost function has been minimized considering reliability constraint in prevailing UC formulation. The UC scheduling is accomplished in short-term so that the reliability is maintained in acceptable level. Because of complex nature of RCUC problem and full AC load flow constraints, the hybrid algorithm included Simulated Annealing (SA) and Binary Particle Swarm Optimization (BPSO) has been proposed to optimize the problem. Numerical results demonstrate the effectiveness of the proposed method and considerable efficacy of the time-based DR program in reducing operational costs by implementing it on IEEE-RTS79.
\end{abstract}

Copyright $@ 2018$ Institute of Advanced Engineering and Science. All rights reserved.

\section{Corresponding Author:}

Masoud Aliakbari,

Department of Electrical and Computer Engineering,

Islamic Azad university,

University campus, Blvd. Shahid Fahmidah, Blvd. Shahid Beheshti, Saveh, Iran.

Email: m.aliakbari.365@gmail.com

\section{NOMENCLATURES}

1) Indices

$a, b, t \quad$ Hours counters at DR and RCUC $\omega_{x}$

$n \quad$ Units counter $\quad \rho(a)$

$i, j \quad$ Bus indices in Load Flow equation $\quad \rho_{0}(a)$

$k \quad$ State of COPT

$x \quad$ Particles counter in PSO

it Iteration

2) Variables

$C_{n}\left(P G_{n, t}\right) \quad$ Cost function of generation unit $\mathrm{n}$

$c_{1} \& c_{2} \quad$ Cognitive and social learning rates
3) Parameters

$C S C_{n}$

$D_{t}$

$D R_{n}$

$E(a, a)$

$E(a, b)$
The inertial of $x^{\text {th }}$ particle

Electricity price in $\mathrm{a}^{\text {th }}$ hour after DR (\$/MWh)

Electricity price in $\mathrm{a}^{\text {th }}$ hour before DR (\$/MWh)

Cold start-up cost of unit $n$

Load demand at time $\mathrm{t}$

Down rate of $\mathrm{n}^{\text {th }}$ unit per hour

Self-elasticity

Cross elasticity of the demand between $\mathrm{ab}^{\text {th }}$ hours 


\begin{tabular}{|c|c|c|c|}
\hline$d(a)$ & $\begin{array}{l}\text { Customer demand in ath hour after } \\
\text { DR (MWh) }\end{array}$ & $H S C_{n}$ & Hot start-up cost of unit $n$ \\
\hline$d_{0}(a)$ & $\begin{array}{l}\text { Customer demand in ath hour } \\
\text { before DR (MWh) }\end{array}$ & $M U T$ & Minimum up time \\
\hline gbest & $\begin{array}{l}\text { The best obtained value of } \\
\text { objective function over PSO } \\
\text { procedure }\end{array}$ & $M D T$ & Minimum down time \\
\hline$I_{n, t}$ & Status of unit $\mathrm{n}$ at hour $\mathrm{t}$ & $N_{b}$ & Number of buses \\
\hline $\operatorname{Loss}_{t}$ & Active power loss of the system & $N_{g}$ & Number of generators \\
\hline$P D G_{n, t}$ & $\begin{array}{l}\text { Output active power of DG unit } n \\
\text { at hour } t\end{array}$ & $N_{D G}$ & Number of DG units \\
\hline$P G_{n, t}$ & $\begin{array}{l}\text { Output active power of generator } n \\
\text { at hour } t\end{array}$ & $P a_{\max }$ & Total number of particles \\
\hline pbest $_{x}$ & $\begin{array}{l}\text { The best previous position of the } \\
\mathrm{x}^{\text {th }} \text { particle }\end{array}$ & $Q_{G, n}^{\max } \& Q_{G, n}^{\min }$ & $\begin{array}{l}\text { Maximum and minimum reactive power } \\
\text { generation limit of unit } \mathrm{n}\end{array}$ \\
\hline$P(\Delta E)$ & $\begin{array}{l}\text { The probability of worse answer } \\
\text { accepting }\end{array}$ & $Q_{D G, n}^{\max } \& Q_{D G, n}^{\min }$ & $\begin{array}{l}\text { Maximum and minimum reactive power } \\
\text { generation DG limit of unit } n\end{array}$ \\
\hline$S_{i j, t}$ & Power flow on ijth line & $S_{i, j}^{\max }$ & Maximum apparent power flow for $\mathrm{ij}^{\text {th }}$ line \\
\hline$S R_{t}$ & Spinning reserve at hour $\mathrm{t}$ & $S C_{n, t}$ & Startup cost of unit $\mathrm{n}$ at hour $\mathrm{t}$ \\
\hline$T_{n, t}^{o f f}$ & $\begin{array}{l}\text { Duration of continuously off state } \\
\text { of unit } n \text { at hour } t\end{array}$ & $T$ & Scheduling period \\
\hline$T_{n, t}^{o n}$ & $\begin{array}{l}\text { Duration of continuously on state } \\
\text { of unit } n \text { at hour } t\end{array}$ & $T_{0}$ & Initial temperature of SA \\
\hline$V_{j}$ & Voltage magnitude at busj & $T_{f}$ & The step for reducing temperature \\
\hline$v_{x}^{i t}$ & $\begin{array}{l}\text { Velocity of } x^{\text {th }} \text { particle in it }{ }^{\text {th }} \\
\text { iteration }\end{array}$ & $U R_{n}$ & Up rate of $n^{\text {th }}$ unit per hour \\
\hline$x_{x}^{i t}$ & $\begin{array}{l}\text { Position of } x^{\text {th }} \text { particle in it }{ }^{\text {th }} \\
\text { iteration }\end{array}$ & $V_{j}^{\max } \& V_{j}^{\min }$ & $\begin{array}{l}\text { Maximum and minimum limits for voltage } \\
\text { magnitude at bus } j\end{array}$ \\
\hline$X_{j}$ & New trial solution of particle & VOLL & Value of lost load \\
\hline$X_{i}$ & Current trial solution of particle & $Y_{i j}$ & $\mathrm{ij}^{\text {th }}$ array of admittance matrix \\
\hline$\theta_{j}$ & Voltage angle of bus $\mathrm{j}$ & $\tau$ & Searching intensifier parameter \\
\hline$\varphi_{i j}$ & $\begin{array}{l}\text { Angle of } \mathrm{ij}^{\text {th }} \text { array of admittance } \\
\text { matrix }\end{array}$ & $\delta_{j}^{\max } \& \delta_{j}^{\min }$ & $\begin{array}{l}\text { Upper and lower voltage angle limits of } \\
\text { bus } j\end{array}$ \\
\hline
\end{tabular}

\section{INTRODUCTION}

In restructured and competitive environment, it is necessary to supply the electrical demand in continuous, reliable and economic manner. Unit commitment is one of the available measures to reach the mentioned goal. In UC procedure, thermal generating units are scheduled to generate power at minimum operation cost so that all constraints of the system and spinning reserve requirements are satisfied. In power system operations, reliability is one of the most important challenges which cannot be neglected by the system operator. Huge investment costs in large power plant and the wasted power in the form of power losses have caused remarkable participation of Distributed Energy Resources (DERs) in power grid configuration. By rapid development of emerging new technologies, the reliability issues have highlighted more than before. A large number of investigations considering the variety type of UC problem have been performed.

The state-transition formulation for the unit commitment (UC) problem is presented in [1] with new decision variables instead of generator's on/off statuses. The integration of solar energy resources into the conventional unit commitment is proposed in [2] as an alternative energy resources using ant lion optimizer with consideration of uncertainties. In [3], the UC problem has been formulated by integrating wind power generators along with thermal power system using grey wolf optimization algorithm as the main optimization tool. Authors in [4] focused on solving efficiently unit commitment of the interconnected multi-site CHP system to coordinate heat and power production in each site using relaxed ON/OFF state based dynamic programming. A contingency-constrained UC is solved in [5] with considering a set of contingency probability distributions Instead of assigning a probability estimate for each contingency scenario. Considering the widespread variety of resources in microgrids, a multi objective UC is carried out in [6] to minimize the operation cost and $\mathrm{CO}_{2}$ emission. Authors in [7] propose an $\mathrm{N}-1$ security constrained formulation for SCUC based on the line outage distribution Factors instead of the conventional method based 
on Injection Sensitivity Factors. A reformulation of the scenario-based two-stage unit commitment problem under uncertainty is provided in [8]. A novel heuristic algorithm called the binary artificial sheep algorithm has been used to solve the UC problem in [9]. In [10] a novel branch-and-cut algorithm using atemporally decomposed bilevel separation aracle is proposed to solve very large scale UC problem. To guarantee system security and reliability, authors in [11] have been developed sufficient dispatchable generation and transmission capacities to improve system operational performance by optimizing schedules considering uncertainty of wind power. Authors in [12] have been recommended a robust RCUC at presence of multiple DERs for best resource allocation and reliability maintaining in a predefined range. Large number of studies regarding DERs management in smart grid has been carried out in recent years that some of them could be briefly surveyed as follows.

A methodology to obtain the optimal location for the placement of multiple DG sources in a distribution network is proposed in [13] by evaluating a global multi-objective technical index using artificial immune system. The DR program is used to optimize the energy profile for commercial sector in [14] with reforming the Maximum Demand from peak zone to off-peak zone to reduce electricity cost using Evolutionary Algorithm. Authors in [15] present the utilization of demand response in the day-ahead operation of a micro-grid in the presence of renewable energy sources. Different DR programs modeling are proposed in [16] and [17] to increase in efficiency of energy consumption with minimum operational costs and desirable quality.

To the best of our knowledge, the impact of time-based DR program in UC procedure has been rarely studied and investigated. So, in this paper, the RCUC is scheduled at presence of DGs and time-based DR program with full AC load flow constraints using a hybrid method. The main contribution of this paper includes incorporating the time-based DR program in UC problem and utilizing a hybrid method to optimize the RCUC Schedule. In order to illustrate the effectiveness of the proposed approach, it is applied to IEEE Reliability Test System (IEEE-RTS79). Finally, a comparison is done between the proposed optimization algorithm and some huristic algorithms. The remainder of this paper is structured as follows. Section 2 briefly discusses the overture of DR programs. Reliability frameworks are presented in section 3. Section 4 discusses the proposed optimization algorithm and the objective function formulation. Section 5 represents the numerical studies and analysis of obtained answers and Section 6 concludes the paper.

\section{DR PROGRAM AND ECONOMIC MODEL OF DEMAND}

DR programs are divided into some main categories and some sub-categories based on Federal Energy Regulatory Commission (FERC) classification [18]. In this work, Time-Of-Use (TOU) method that is one of the time-based programs is employed. TOU can shift some of the unnecessary demand from peak load period to low load period. This offset is because of customer's demand sensitivity with respect to electricity price changes at different periods. A conservative model of load has been extracted as response of elastic demand against the price signals expounded in [19]. The mentioned economic model (power model) is composed from two concepts, single period and multi period loads which are so called "self-elasticity" and "cross elasticity", respectively. The loads with self-elasticity cannot shift from one period to another (e.g. lighting loads) while the loads with cross elasticity can shift from one period to others. In this paper, to avoid restatement of modeling procedure, final economic models of each concept (self and cross elasticity) of responsive loads are given in (1) and (2), respectively.

$$
\begin{aligned}
& d(a)=d_{0}(a) \cdot\left(\frac{\rho(a)}{\rho_{0}(a)}\right)^{E(a, a)} \\
& d(a)=d_{0}(a) \cdot\left(\frac{\rho(b)}{\rho_{0}(b)}\right)^{E(a, b)} \\
& d(a)=d_{0}(\boldsymbol{a}) \cdot \prod_{\substack{b=\mathbf{1} \\
\boldsymbol{b} \neq \boldsymbol{a}}}^{\mathbf{2 4}}\left(\frac{\rho(b)}{\rho_{0}(b)}\right)^{E(a, b)}
\end{aligned}
$$

Each load point comprises a combination of two mentioned type of loads in the power system. So, composite period elastic load model which is presented in (3) is employed to implement the TOU program, in this paper. 


\section{RELIABILITY EVALUATION}

\subsection{Availability of generating units}

In operation phase, the probability of generation unit supplying ability or inability is based on the Lead Time (LT). At this time interval, the surplus units can not commit or replace with committed units in increasing load states or lose some power production. The probability of state which the generating unit fails in LT interval is recognized as Outage Replacement Rate (ORR) of each unit. In reliability analysis, each generating unit could be either available or unavailable as a two state model represented in Figure 1.

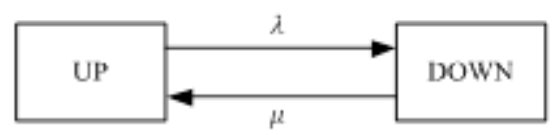

Figure 1. Two state model of each generating unit

If the failure and repair rate is distributed as exponential functions, the probability of unavailability of each unit will be given as (4).

$$
U(L T)=\frac{\lambda}{\lambda+\mu}-\frac{\lambda}{\lambda+\mu} \cdot e^{-(\lambda+\mu) \cdot T}
$$

Where, $\lambda$ and $\mu$ are the failure and repair rate of any generating unit, respectively. Neglecting the repairing procedure in operating phase, (4) is modified as (5).

$$
U(L T)=1-e^{-\lambda T}
$$

\subsection{Calculation of reliability indices}

Two of the most important indices in reliability studies are loss of load probability (LOLP) and Expected Energy Not Supplied (EENS). The LOLP is the system risk index and the EENS expresses the expected energy that will not be served by power system in corresponding period. In this paper, to calculate the reliability indices the concept of the convolution of the capacity outage probability table (COPT) expounded in [20] is applied. The aforementioned indices are calculated through COPT as (6)-(9). In (6)-(8), $C R_{k, t}$ and $P R_{k, t}$ are total capacity remaining in service of state $\mathrm{k}$ of COPT and the corresponding probability respectively.

$$
\begin{aligned}
& \operatorname{LOSS}_{k, t}=\left\{\begin{array}{l}
1, \text { if } \quad C R_{k, t}<D_{t} \\
0, \text { otherwise }
\end{array} \quad t \in[1, T]\right. \\
& L O L P_{t}=\sum_{k=1}^{K} P R_{k, t} \cdot \operatorname{LOSS}_{k, t} \quad t \in[1, T] \\
& \operatorname{EENS}_{t}=\sum_{k=1}^{K} P R_{k, t} \cdot \operatorname{LOSS}_{k, t} \cdot\left(D_{t}-C R_{k, t}\right) \quad t \in[1, T] \\
& \text { EENS }_{T o t}=\sum_{t=1}^{T} E_{E N S_{t}} \quad t \in[1, T]
\end{aligned}
$$

\section{METHODOLOGY}

The UC is one of the most difficult optimizing problems and lots of constraints must be considered. The main purpose of this scheduling is minimizing the operation cost during dispatch period. Considering the complication of UC problem, involving the evolutional methods in unit scheduling is unavoidable especially when reliability constraints and AC load flow constraints are addressed.

\subsection{Simulated annealing}

Simulated Annealing (SA) proposed by Metropolis at 1957 in order to handle optimization problems with a large searching space [21]. SA at a common form has been presented based on the similarity between the cooling molten metal and solving the combinatorial optimization problems. If the temperature of the molten metal is gradually reduced, all atoms can regulate themselves in their lowest energy level. This algorithm can accept worse answers to escape from local optimum trapping in different situations. It is started with an initial stochastic state and by a transition from one state to another (neighboring state) 
gradually minimizes the cost function. In each trial, the current answer is compared with previous configuration answer as (10).

$$
\Delta E=\left(X_{j}-X_{i}\right) / X_{i}
$$

The value of $\Delta \mathrm{E}$ can be either positive, negative or zero. If the new obtained solution is better than the current one (negative value of $\Delta \mathrm{E}$ ), it will be accepted and otherwise (zero or positive value of $\Delta \mathrm{E}$ ) the acceptance is performed based on a probability given by Boltzmann distribution as follows:

$$
\begin{aligned}
& P(\Delta E)=\exp \left(-\Delta E / \text { Temp }_{i t}\right) \\
& \operatorname{Temp}_{i t} \begin{cases}T_{0} & \text { it }=1 \\
\text { Temp }_{i t-1} \times T_{f}, & \text { it }>1\end{cases}
\end{aligned}
$$

\subsection{Binary particle swarm optimization}

Binary Particle Swarm Optimization (BPSO) is an intelligent population-based algorithm which can search the discrete space to solve optimization problems. Decision variables in this method are " 0 " or " 1 " bits of a binary string. Several version of this algorithm based on the problem modality has been extended. An improved version of binary PSO algorithm has been utilized in this paper expounded in [22]. In any iteration the position of particle is updated as follows:

$$
\begin{aligned}
& x_{x}^{i t} \leftarrow x_{x}^{i t-1} \oplus v_{x}^{i t} \quad x=1,2, \ldots, P a_{\max } \\
& v_{x}^{i t}=\bar{\omega}_{x}^{i t-1}+\omega_{x}^{i t-1} \cdot\left(c_{1 x} \cdot\left(\text { pbest }_{x}^{i t-1} \oplus x_{x}^{i t-1}\right)+c_{2 x} \cdot\left(\text { gbest }^{i t-1} \oplus x_{x}^{i t-1}\right)\right) \\
& P \omega^{i t}=P \omega_{\max } \cdot e^{-\lambda_{P} \cdot(i t-1)} \quad i t=1,2, \ldots, \text { Max It } \\
& \lambda_{P}=\frac{\ln \left(\frac{P \omega_{\max }}{P \omega_{\min }}\right)}{(\text { MaxIt }-1)^{\tau}}
\end{aligned}
$$

Equation (14) shows the velocity updating procedure at it ${ }^{\text {th }}$ iteration based on the previous iteration. It should be noted here that parameter $\mathrm{P} \omega$ plays an important role in the algorithm progress. In primary iterations, the value of inertial probability $P \omega$ is high and the algorithm explores the search space. Gradually, by increasing the number of iterations and decreasing the $P \omega$ value, the exploration is converted to exploit the search space with small changes in variables.

\subsection{Optimization procedure}

In spite of the excellent capabilities of PSO algorithms in exploring the feasible region and fast convergence, there is a considerable probability for trapping in local optimums in algorithm. This defect has been somewhat eliminated with SA method by defining a probability for accepting the worse solutions. If this advantage is synthesized into PSO structure, the loosing information on local optimums is decelerated while the performance is increased. In this regard, an improved version of binary PSO algorithm recommended in [22] is taken into consideration as main algorithm. The BPSO is updated in two stages that so called "personal best update" and "Global best update". In this paper, the personal best update procedure of each particle is done based on SA algorithm at any iteration. As, if new obtained solution of particle at it ${ }^{\text {th }}$ iteration was better than of its current personal best then the solution is accepted as personal best (personal best updating) and otherwise the acceptance of solution as personal best is done based on probability given by (10). Also, the global best is normally updated at any iteration and it is considered as final solution at last iteration eventually. This combination assures the acceleration in convergence and increases the ability in deep search process. In this paper, a hybrid SA and BPSO is proposed for combining capabilities of these methods and eliminating their weaknesses. The flowchart of the proposed hybrid algorithm is illustrated in Figure 2. 


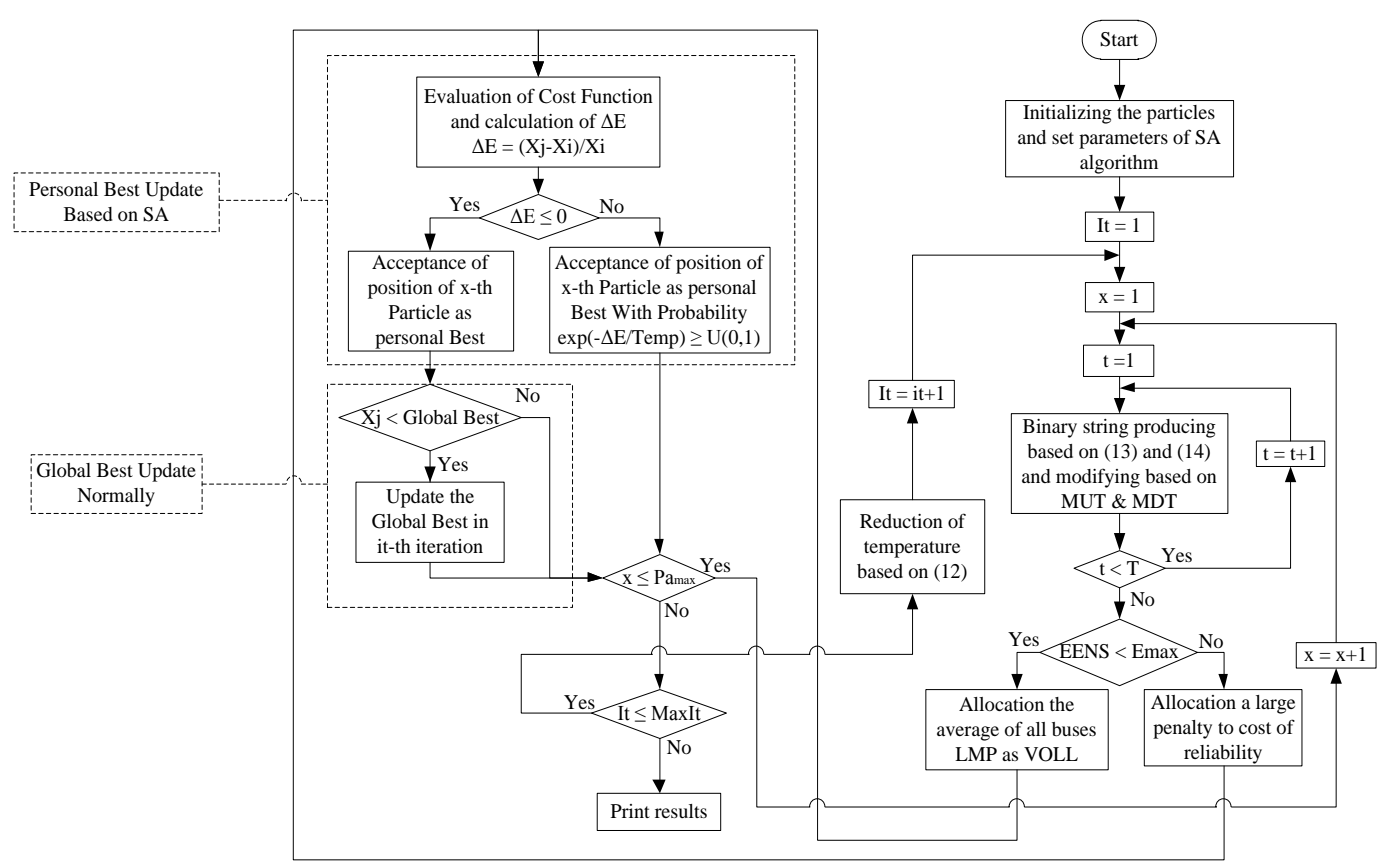

Figure 2. Flow chart of the proposed algorithm

\subsection{Objective function and constraints}

Some of involved constraints in RCUC implementing are presented in this section. Fuel cost of each thermal unit is a second order function of its generated power which is expressed as follows:

$$
\mathcal{F C}\left(P G_{n}\right)=\alpha_{n}+\beta_{n} P G_{n}+\gamma_{n}\left(P G_{n}\right)^{2}
$$

In (17), $\alpha_{n}, \beta_{n}$ and $\gamma_{n}$ are fuel cost coefficients. The objective function of UC problem in the presence of DG units and DR program with the aim of decreasing operation and reliability cost is formulated as (18). The mentioned objective function consists of two terms. The first term represents fuel cost of conventional and DG units together with starting up cost to meet the demand. The hourly cost of EENS has been shown in second term. The developed objective function in (18) is subjected to some constraints as follows:

$$
\min \sum_{t=1}^{T} \sum_{n=1}^{N_{G}+N_{D G}}\left[\mathcal{F C}\left(P G_{n, t}\right) I_{n, t}+S C_{n, t}\right]+C_{E E N S}\left(E E N S_{t}\right)
$$

Equation (19) is the reliability constraint and its cost is obtained by (20). The power balance and spinning reserve constraints are represented in (21) and (22). The constraints (23)-(28) ensure the proper operation of generation units considering the power flow limits. Startup cost can be evaluated based on (29) and also it can be considered as a constant value for each generation unit. Constraints on minimum up time, minimum down time and ramp rates of generation units are given through (30)-(33), respectively. Network security constraints are represented in (34) and (35).

$$
\begin{aligned}
& E_{\text {ENS }} \text { Tot } \leq E_{\text {max }} \times \text { Total Energy } \\
& C_{E E N S}\left(E E N S_{t}\right)=E E N S_{t} \times \text { VOLL }_{t} \\
& \sum_{n=1}^{N_{G}+N_{D G}} P G_{n, t}=D_{t}+\text { LosS }_{t} \pm D_{T O U, t} \\
& \sum_{n=1}^{N_{G}+N_{D G}} P G_{n}^{\max } \times I_{n, t} \geq S R_{t}+D_{t}+\text { LosS }_{t} \\
& P G_{i}-P L_{i}=\sum_{j=1}^{N} V_{j} \times\left|Y_{i j}\right| \times \cos \left(\theta_{i}-\theta_{j}-\varphi_{i j}\right) \\
& Q G_{i}-Q L_{i}=\sum_{j=1}^{N} V_{j} \times\left|Y_{i j}\right| \times \sin \left(\theta_{i}-\theta_{j}-\varphi_{i j}\right)
\end{aligned}
$$




$$
\begin{aligned}
& P G_{n}^{\min } \leq P G_{n} \leq P G_{n}^{\max } \\
& P_{D G, n}^{\min } \leq P_{D G, n} \leq P_{D G, n}^{\max } \\
& Q G_{n}^{\min } \leq Q G_{n} \leq Q G_{n}^{\max } \\
& Q_{D G, n}^{\min } \leq Q_{D G, n} \leq Q_{D G, n}^{\max } \\
& S C_{n}=\left\{\begin{array}{lll}
H S C_{n} & \text { if } & M D T_{n} \leq T_{n}^{\text {off }} \leq M D T_{n}+C S H_{n} \\
C S C_{n} & \text { if } & T_{n}^{\text {off }}>M D T_{n}+C S H_{n}
\end{array}\right.
\end{aligned}
$$

Where

$$
\begin{aligned}
& H_{n}^{\text {off }}=M D T_{n}+C S H_{n} \\
& \left(T_{n, t-1}^{\text {off }}-M D T_{n}\right)\left(I_{n, t-1}-I_{n, t}\right) \geq 0 \\
& \left(T_{n, t-1}^{\text {on }}-M U T_{n}\right)\left(I_{n, t-1}-I_{n, t}\right) \geq 0 \\
& P G_{n, t}-P G_{n, t-1} \leq U R_{n} \\
& P G_{n, t-1}-P G_{n, t} \leq D R_{n} \\
& V_{j}^{\text {min }} \leq V_{j} \leq V_{j}^{\text {max }} \\
& \left|S_{i j, t}\right| \leq S_{i j}^{\text {max }}
\end{aligned}
$$

\section{NUMERICAL STUDY}

The RCUC incorporated with DGs and DR implementation is carried out over the IEEE-RTS79 [23]. It is assumed that three $25 \mathrm{MW}$ small scale gas turbines as DGs are located in 3, 9, and 10 buses. The hourly AC optimal power flow (ACOPF) is performed utilizing MATPOWER toolbox in MATLAB software to distribute the generated power considering the network constraints [24]. The MATPOWER toolbox employs MATLAB Interior Point Solver (MIPS) for ACOPF. The specifications of DGs for operation cost and reliability assessment is extracted from [14]. Also, the common information related to conventional units in UC problem (e.g. starting up and shutting down costs, ramp rate and etc.) have been extracted from [25]. The minimum spinning reserve requirement is assumed to be $10 \%$ of hourly demand. DR programs are also investigated based on TOU model which is one of the time-based DR programs. In order to implement TOU program, a typical load curve has been extracted from [19] and were normalized based on peak hour load $(2670 \mathrm{MW})$ in this case study. The load curve is divided into three intervals: low load period (02:00 to 09:00), off-peak period (10:00 to 19:00) and peak period (20:00 to 01:00). The elasticity of the loads is shown in Table 1 which is originally taken from [19].

Table 1. The Elasticity of Loads for DR Implementing

\begin{tabular}{cccc}
\hline & Peak & Off-Peak & Low-Peak \\
\hline Peak & -0.2 & 0.016 & 0.012 \\
Off-Peak & 0.006 & -0.2 & 0.01 \\
Low-Peak & 0.008 & 0.01 & -0.2 \\
\hline
\end{tabular}

Two scenarios are discussed in numerical studies. In the first scenario, operation without DGs and DR program is presented. Then, the new load curve is obtained by TOU implementing on main load curve at any bus using first scenario Local Marginal Prices (LMPs). In the second scenario, DGs and conventional units are committed in RCUC at presence of new load curve all over the network. The base prices at each load bus are equal to average LMPs for 24 hours derived from OPF in the first scenario. The ratio of electricity prices has been considered one half of base prices in low load period, equal to base prices in off-peak and equal to one and a half times of the base prices at peak period [26]. Participation potential for DR implementation is assumed to be $20 \%$ at any load point. The acceptable level of reliability is a predefined percentage of consumption energy which the maximum allowable value has been considered to be $0.1 \%$ of daily consumed 
energy in this study. The reliability evaluating is based on HL-I. The reliability constraint is applied to each feasible solution of particles as follows:

If $\mathrm{EENS}_{\mathrm{Tot}}<\mathrm{E}_{\max }$, the cost of reliability is calculated based on average of all buses LMPs at any hour.

If $\mathrm{EENS}_{\mathrm{Tot}}>\mathrm{E}_{\max }$, the trial solution provides an undesired level of reliability and a fixed large penalty is allocated to reliability cost in the second term of the cost function.

Final solution is chosen through several trials considering the stochastic inherent behavior of evolutionary algorithm. Parameters setting of proposed algorithm have been determined based on trials which are presented in Table 2. The optimal operation of DG units and demand variations due to TOU implementation are presented in Figure 3.

Table 2. Setting Parameters for Proposed Algorithm

\begin{tabular}{ccccccc}
\hline$P \omega_{\min }$ & $P \omega_{\max }$ & MaxIt & $P a_{\max }$ & $T_{f}$ & $T_{0}$ & $\tau$ \\
\hline 0.05 & 0.95 & 200 & 40 & 0.98 & 10 & 0.2 \\
\hline
\end{tabular}

As it can be seen in Figure 3, the price signals from TOU can considerably alter the demand and reduce the peak demand by $186 \mathrm{MW}$. The hourly DGs participation is obtained by proposed hybrid algorithm decision beside other conventional units. With increase in load at peak period, DGs participation is increased and injected power by small non-expensive units affects the most significant operational indices. Hence, an extensive numerical comparison has been presented in Table 3 while the lead time was fixed at $4 \mathrm{~h}$. With increase in power injections by DGs, de-committing expensive generators and altering demand profile, relief of transmission capacity and decrease of operational costs could be achieved simultaneously. The TOU implementation can render some demand from peak load period to low load period to prevent load shedding and system voltage collapse when the power system reliability is jeopardized. The initial load forecasting and the load curve after applying TOU have been shown in Figure 4. The optimal unit commitment scheduling results for both scenario are shown in Table 4 and Table 5.

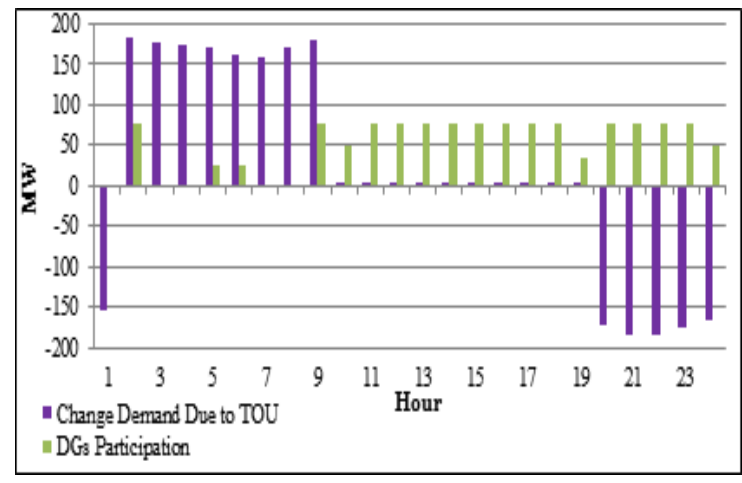

Figure 3. The injected power by DGs and the change demand due to TOU for LT $=4 \mathrm{~h}$

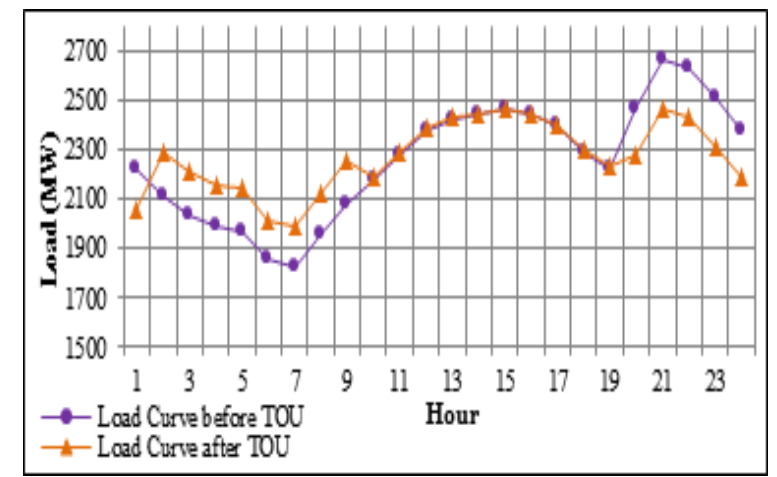

Figure 4. Load curve before and after TOU implementing 
Table 4. Optimal Unit Commitment Schedule Results without DG \& DR

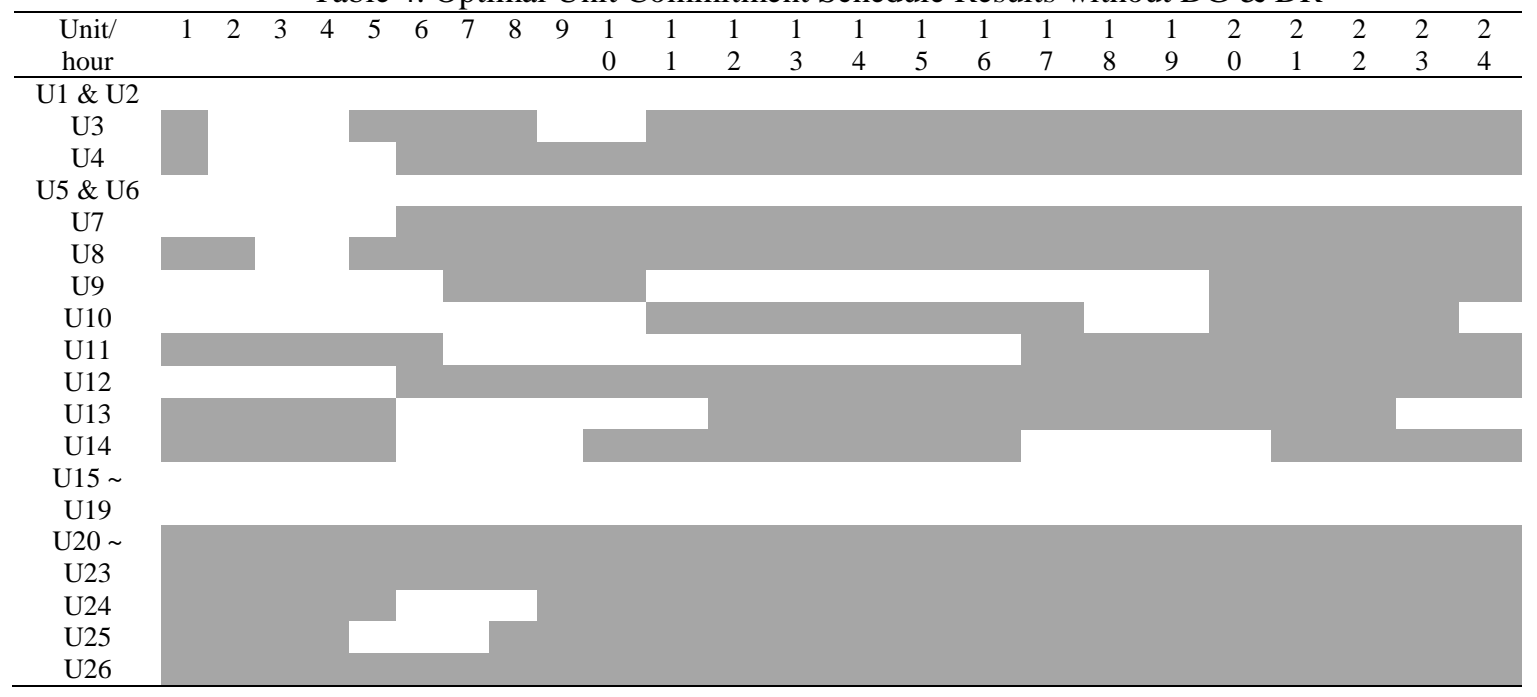

Table 5. Optimal Unit Commitment Schedule Results with DG \& DR

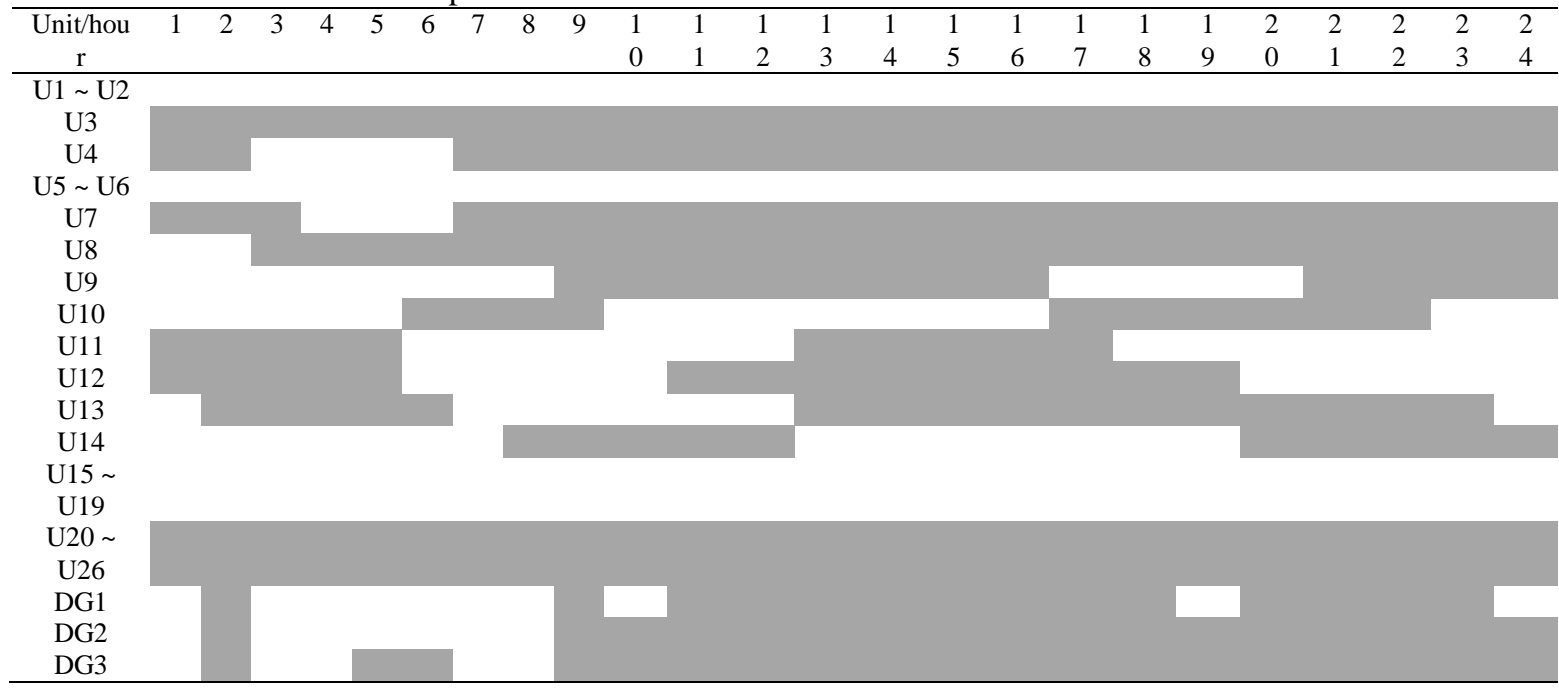

Achieving a better level of reliability requires the commitment of more units and this increases the RCUC cost in dispatch period. However results of Table 3 shows that a better reliability index can be obtained with lower operational costs by optimal scheduling of generation units. The improvement of considered reliability index (EENS) demonstrates the applicability and usefulness of combining the DGs and DR program and proposed algorithm. Lead time is another parameter that affects the reliability indices. The LOLP index for different amount of lead time has been presented in Figure 5. This index indicates the probability of state that the available generation along with spinning reserve is not able to meet the system load and satisfy the constraints.

Table 3. Comparison between Several Operational Parameters (LT=4 h)

\begin{tabular}{lcc}
\hline \multicolumn{1}{c}{ Operational Parameters } & With DG \& DR & Without DG \& DR \\
\hline Total cost of RCUC (\$) & $963,145.97$ & $1,043,806.94$ \\
Start Up Cost (\$) & 23,000 & 28,500 \\
Cost of conventional units (\$) & $913,768.28$ & $1,011,038.87$ \\
Cost of DGs (\$) & $25,309.53$ & 0 \\
Cost of reliability (\$) & $1,068.16$ & $4,268.07$ \\
Operation Cost reducing due to TOU (\$) & $52,351.44$ & 0 \\
Transmission Network Congestion at peak hour (MW) & $3,413.73$ & $3,607.4$ \\
Active power Loss (MW) & 846.01 & 830.64 \\
Load Factor (\%) & 91.57 & 84.61 \\
EENS (MWh/day) & 24.09 & 28.31 \\
\hline
\end{tabular}


Figure 5 depicts that the lead time can affect the solution of UC problem. The change in lead time results in change of generating unit unavailability and this issue causes an increase or decrease in number of committed units to provide the same reliability level. In order to demonstrate the effect of lead time variations, Figure 6 and Figure 7 illustrate the total committed capacity of each load curve shown in Figure 4 while the lead time is varied from 4 to $8 \mathrm{~h}$. It can be seen that the required spinning reserve grows with increase in committed capacity to achieve the same desired level of reliability.

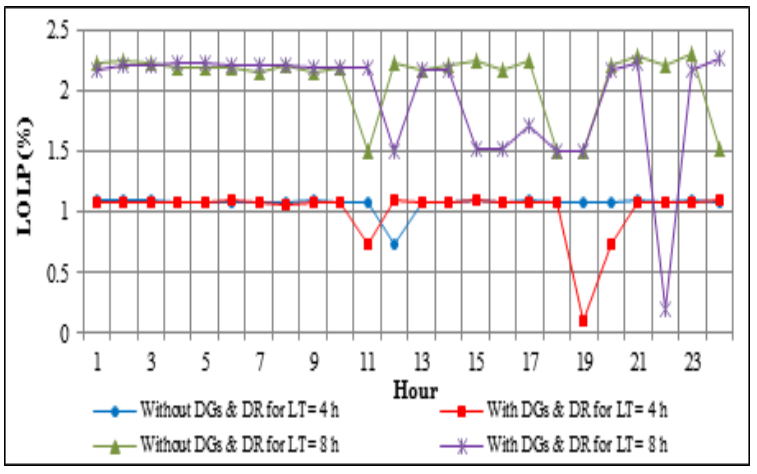

Figure 5. LOLP at each hour over the dispatch period

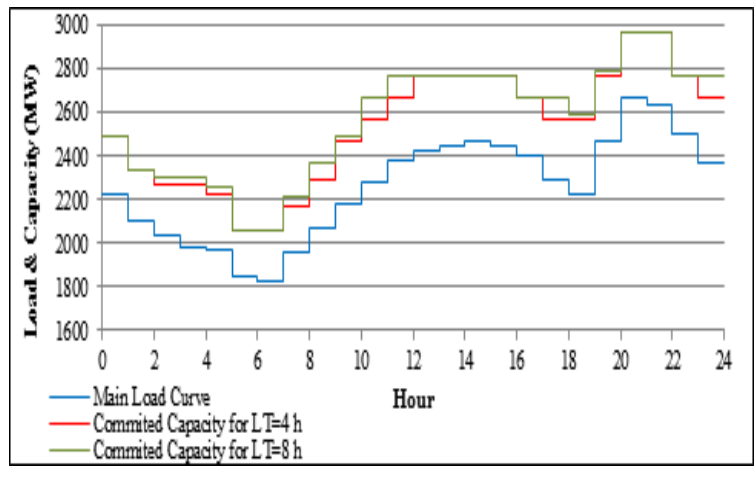

Figure 6. Expected initial load and total committed capacity at each hour for two values of lead time

To validate the computational efficiency of the proposed hybrid method, the same formulation is solved using binary real coded firefly (BRCFF) algorithm and novel adaptive quantum-inspired binary gravitational search (QBGSA) algorithm. The considered algorithms have the ability for search in large space with fast convergence rate. A more detailed description of this algorithms, including formulating and flowchart of optimizing procedure can be found in [27] and [28]. Figure 8 depicts comparison between proposed optimization method, QBGSA, BPSO and BRCFF based on RCUC formulation to assess how searching the space of problem. High gap between convergence points of algorithms reveal the powerfulness of the proposed method against other ones. Considering the obtained results proposed algoritm provides the best optimal solution in terms of computation speed and accuracy compared with others. The objective of this comparison is to select the most appropriate optimization technique for solving the UC problem in future research.

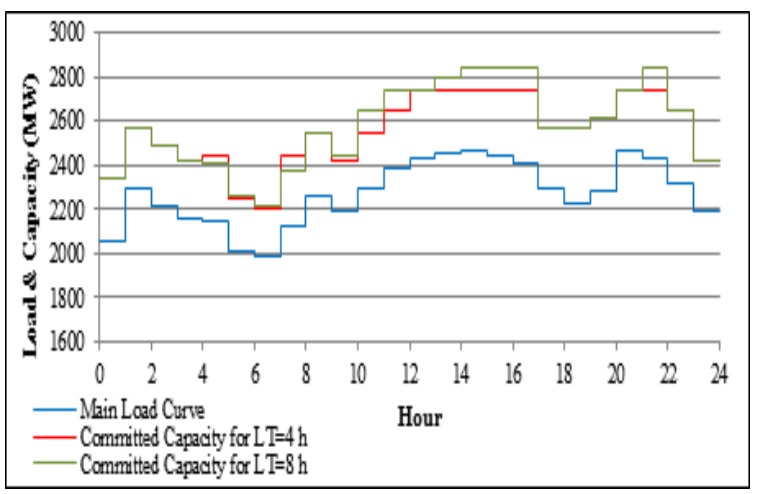

Figure 7. Load curve after TOU and total committed capacity at each hour for two values of lead time

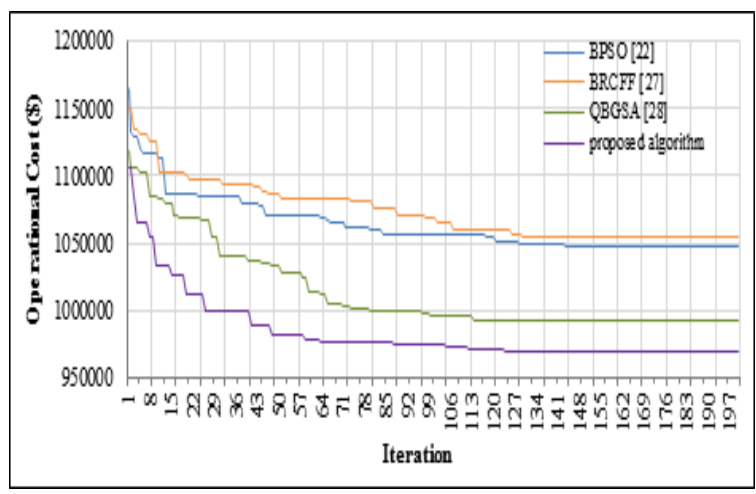

Figure 8. Convergence characteristics of proposed method, QBGSA, BPSO and BRCFF (LT=4 h)

\section{CONCLUSION}

In this paper, a new approach which is a combination of two heuristic algorithms was presented to solve the UC problem at presence of DGs and DR programs with reliability and AC power flow constraints. The enforcement of reliability constraint determines the specific level of generation and committed capacity 
to maintain the reliability in acceptable range. Numerical and graphical results prove the powerfulness of proposed hybrid algorithm and illustrate that the time-based DR program integrated with small nonexpensive DG units is able to lead to a more reliable and further economic operational condition. Remarkable improvement of reliability indices and operational efficiency simultaneously will be achievable by accurate hourly DG allocating at presence of DR program with negligible cost. Considering the large number of accomplished work in context of UC scheduling, there is a need for further investigation in this area. Accordingly, employing the several types of DR programs by exactly modeling the participated customer's behavior can lead to more realistic results.

\section{REFERENCES}

[1] S. Atakan, G. Lulli, S. Sen, "A State Transition MIP Formulation for the Unit Commitment Problem," IEEE Transaction on Power System, vol. 33, no. 1, pp. 736-749, 2018.

[2] I. Nadhirah, Z. Mat, Y, Z. Zakaria," Ant Lion Optimizer for Solving Unit Commitment Problem in Smart Grid System", Indonesian Journal of Electrical Engineering and Computer Science (IJEECS), vol. 8, no. 1, pp. 129-136, October 2017.

[3] S. S. Sakthi, R. K. Santhi, N. M. Krishnan, et. al, "Wind Integrated Thermal Unit Commitment Solution using Grey Wolf Optimizer", International Journal of Electrical and Computer Engineering, vol. 7, no. 5, pp. 2309-2320, 2017.

[4] A. Rong, P. B. Luh, "A Dynamic Regrouping Based Dynamic Programming Approach for Unit Commitment of the Transmission-constrained Multi-site Combined Heat and Power System”, IEEE Transaction on Power System, pp. 1-9, 2017.

[5] C. Zhao, R. Jiang, "Distributionally Robust Contingency-Constrained Unit Commitment", IEEE Transaction on Power System, pp. 1-9, 2017.

[6] C. Deckmyn, J. V. de Vyver, T. L. Vandoorn, B. Meersman, J. Desmet, L. Vandevelde, "Day-ahead unit commitment model for microgrids", IET Generation, Transmission \& Distribution, pp. 1-9, 2016.

[7] D. A. Tejada-Arango, P. Sánchez-Martın, A. Ramos, "Security-constrained unit commitment Line Outage Distribution Factors", IEEE Transaction on Power System, pp.1-9, 2017.

[8] I. Blanco, J. M. Morales, "An Efficient Robust Solution to the Two-Stage Stochastic Unit Commitment Problem," IEEE Transaction on Power System, pp. 1-11, 2017.

[9] W. Wang, C. Li, X. Liao, H. Qin, "Study on unit commitment problem considering pumped storage and renewable energy via a novel binary artificial sheep algorithm", Applied Energy, pp. 612-626, 2017.

[10] Z. Guo, R. Li-Yang C. Member, N. Fan, J. P. Watson, "Contingency-Constrained Unit Commitment with Intervening Time for System Adjustments," IEEE Transaction on Power System, pp. 1-11, 2016.

[11] Y. Wang, Sh. Zhao, Zh. Zhou, A. Botterud, Y. Xu, R. Chen, "Risk Adjustable Day-Ahead Unit Commitment with Wind Power Based on ChanceConstrained Goal Programming", IEEE Transactions on Sustainable Energy, pp. 1-11, 2016.

[12] S. Goleijani, T. Ghanbarzadeh, F. S. Nikoo, M. P. Moghaddam, "Reliability constrained unit commitment in smart grid environment”, Electric Power Systems Research, pp.100-108, 2013.

[13] Meera P.S., S. Hemamalini, "Optimal Siting of Distributed Generators in a Distribution Network using Artificial Immune System", International Journal of Electrical and Computer Engineering (IJECE), vol. 7, no. 2, pp. 641-649, April 2017.

[14] M. F. Sulaima, N. Y. Dahlan, et. al, " Optimum Enhance Time of Use (ETOU) for Demand Side Electricity Pricing in Regulated Market: An Implementation Using Evolutionary Algorithm", Indonesian Journal of Electrical Engineering and Computer Science, Vol. 8, No. 1, pp. 253-261, October 2017.

[15] G. R. Aghajani, H. A. Shayanfar, H. Shayeghi, "Presenting a multi-objective generation scheduling model for pricing demand response rate in micro-grid energy management", Energy Conversion and Management, pp. 308-321, 2015.

[16] M. Muratori, G. Rizzoni, "Residential Demand Response: Dynamic Energy Management and Time-Varying Electricity Pricing”, IEEE Transaction on Power System, pp. 1-10, 2015.

[17] K. McKenna, A. Keane, "Residential Load Modeling of Price-Based Demand Response for Network Impact Studies", IEEE Transaction on Smart Grid, pp. 1-10, 2015.

[18] F.E.R.C., "Assessment of Demand Response and Advanced Metering, Staff Report", Federal Energy Regulatory Commission, Washington, DC, 2016.

[19] H. Aalami, G. R. Yousefi, M. P. Moghadam, "Evaluation of nonlinear models for time-based rates demand response programs", Electrical Power and Energy Systems, vol. 65, pp. 282-290, 2015.

[20] R. Billinton and R. N. Allan, 1996, "Reliability evaluation of power systems", Springer, New York: Plenum Press, pp. 150-173, 1996.

[21] S. Kirkpatrick, C. D. Gelatt, Jr., and M. P. Vecchi, "Optimization by simulated annealing”, Science, vol. 220, pp. 671-680, 1983.

[22] M. Gómez, A. López, F. Jurado, "Optimal placement and sizing from standpoint of the investor of Photovoltaics Grid-Connected Systems using Binary Particle Swarm Optimization,” Applied Energy, vol. 87, pp. 1911-1918, 2010.

[23] P. Subcommittee, "IEEE reliability test system", IEEE Transaction Power Apparatus and System, pp. 2047-2054, 1979. 
[24] R. D. Zimmerman, C. E. Murillo-Sanchez, and R. J. Thomas, "Matpower: Steady-state operations, planning and analysis tools for power systems research and education," IEEE Transaction on Power System, vol. 26, no. 1, pp. 12-19, Feb. 2016.

[25] C. Wang, M. Shahidehpour, "Effects of ramp-rates limits on unit commitment and economic dispatch," IEEE Transaction on Power System, vol. 26, no. 1, pp. 1341-1351, 1993.

[26] Iran Ministry of Energy. Statistical information on energy balance. http://www.iranenergy.org.ir; 2016.

[27] K. Chandrasekaran, S. P. Simon, "Network and reliability constrained unit commitment problem using binary real coded firefly algorithm", Electrical Power and Energy Systems, pp. 921-932, 2012.

[28] A. A. Ibrahim, A. Mohamed, H. Shareef, "Optimal power quality monitor placement in power systems using an adaptive quantum-inspired binary gravitational search algorithm", Electrical Power and Energy Systems, pp. 404-413, 2014. 